\title{
Outcome of surgical management of initially irresectable or complicated multiple colorectal liver metastases
}

\author{
H Said, ${ }^{a} M D$; Gamal Fawzy, ${ }^{a} M D ; \boldsymbol{A}$ Kamel, ${ }^{a} M D ;$ K Elhosseny, ${ }^{b} M D$; \\ Ramy Ghali, ${ }^{b} M D$; Mohmad Elgharib, ${ }^{b} M D$; Mohamed Hamdy Attya, ${ }^{c} M D$; \\ A Abdelaal, ${ }^{a} M D ;$ Abdel Wahab Ezzat, ${ }^{a} M D$
}

a) Department of General Surgery, Ain Shams University, Cairo, Egypt.

b) Department of Medical Oncology, Ain Shams University, Cairo, Egypt.

c) Department of Diagnostic Radiology, Ain Shams University, Cairo, Egypt.

d) Department of General Medicine, Ain Shams University, Cairo, Egypt.

\begin{abstract}
Introduction: Hepatic resection is the procedure of choice for curative treatment of colorectal liver metastases (CLM). ${ }^{1}$ Hepatectomy allows five-year survival rates up to $58 \%$ in selected cases $^{2}$ and 10 year survival rates of $16 \% \%^{3}$ to $23 \% .{ }^{4}$ The use of surgical innovations, such as staged resection, portal vein embolization, and repeated resection has allowed higher resection rates in patients with bilobar disease. The use of neoadjuvant chemotherapy allows up to 38\% of patients previously considered irresectable to be significantly downstaged and eligible for hepatic resection. ${ }^{2}$

Design: A prospective study.

Patients: From January 2009 to December 2011, 30 consecutive patients (16 male and 14 female) with multiple colorectal liver metastases (synchronus and metachronus) underwent surgical intervention in Ain Shams University Hospitals. The mean age was 49.9 years $(27$ to $75 y, S D \pm 10.31)$. Simultaneous resection was done when primary lesion was not locally advanced, no intestinal obstruction and the metastases were easily resectable with adequate future liver volume. Neoadjuvant chemotherapy was started in all metachronus and selected patients with synchronus liver metastases. The aim of neoadjuvant was to downstage irresectable tumors and test their biological behavior. Follow up of patients with clinical examination, tumor marker and radiological assessment for a median follow up period of 12 months was done.

Results: This study was conducted on 30 patients. Twenty eight patients had adenocarcinoma (93.3\%), 1 mucinous adenocarcinoma (3.3\%) and 1 liomyosarcoma of the colon (3.3\%). LNS were positive in $76.7 \%$ and negative in $23.3 \%$ of cases. Ninety one colorectal metastatic tumors were identified (synchronous in 9 patients and metachronus in 21 patients) situated in the right lobe, left lobe and bilobar in 53\%, 12\%, and 35\% respectively. CEA was elevated in 14 cases and CA19.9 was elevated in 7 cases. Fifty percent underwent major hepatectomy and 50\% underwent minor hepatectomy. The mean postoperative hospital stay was $9.5 \pm 3.13$ days. Twenty nine cases required postoperative ICU admission and the mean postoperative ICU stay was $1.8 \pm 1.04$ days. Six patients (20\%) had perioperative complications as follows: biliary leak in three cases (10\%), intra-abdominal collection in one case (3\%), chest infection in one case (3\%) and pulmonary embolism in one case (3\%). All these complications were treated conservatively. There were no cases of postoperative liver failure. Nine patients (30\%) had recurrent malignant disease (mean follow up period was $12 \pm 8$ ). Recurrence was local (17\%), hepatic (6\%) or combined local and distant extra-hepatic (6\%). Cases with hepatic recurrence were managed by hepatic resection in one case and the other by percutaneous RFA. Two mortalities were reported. The first patient died from extensive myocardial infarction 3 months postoperatively. The second patient died 15 months postoperatively due to respiratory failure from pulmonary metastases.
\end{abstract}


Conclusion: Surgical resection is the only potentially curative treatment of colorectal metastases. Resectability is no longer restricted and durable survival is possible even in patients with multiple and large metastases. The philosophy is to be more aggressive, tailoring the management plan by multidisciplinary team, and to increase the indications for surgical resection by using one or combination of the following techniques (Portal vein embolization, local ablative techniques, new chemotherapy or staged hepatectomy).

\section{Introduction:}

Colorectal carcinoma with hepatic metastases was long considered as an incurable disease. Recent advances in surgical treatment have substantially improved the affected patients' prognosis. At first, surgery was only performed in patients whose hepatic tumor burden was small. Currently, however, the main issue is the feasibility of curative resection of all metastases. Hepatectomy allows 10 year survival rates of $16 \%{ }^{3}$ to $23 \%{ }^{4}$

\section{Patients and methods: \\ Patient population:}

From January 2009 to December 2011, 30 consecutive patients with multiple colorectal liver metastases (synchronous and metachronus) underwent surgical intervention in Ain Shams University Hospitals. They were 16 male and 14 female patients. The mean age was 49.9 years ( 27 to $75 y, S D \pm 10.31$ ). Patients with poor general condition, extra-hepatic metastases, tumors not responding to downstaging or inability to preserve adequate liver volume with good blood supply, venous and biliary drainage (even after different modalities to increase liver volume e.g. portal vein ligation) were excluded from this study.

\section{Preoperative evaluation:}

Meticulous clinical assessment of general condition was done. Thoracic and pelviabdominal $\mathrm{CT}$ with contrast injection was done to identify the primary tumor (localization, size, spread to neighboring organs, nodal extension), liver metastases (uni- or bilobar, number, size, localization, vascular invasion), presence of lung involvement and detection of any other extra hepatic metastases. When CT was risky (e.g. Iodine allergy) or when CT was normal but there was high suspicion of liver lesion (as increased levels of tumor markers) MRI was performed. Laboratory tests including liver functions, bleeding profile were done. Tumor markers CEA and CA19-9 did not affect the decision but they were important in the follow up and prognosis. Colonoscopy was done for detection of colorectal tumor (primary or recurrence). As a routine it was repeated before hepatic surgery if the last one was done more than 6 months before. CT volumetry was done in cases of aggressive resection plans to detect residual liver volume. Future liver remnant of more than $25 \%$ was accepted but in cirrhotic livers future liver remnant (FLR) more than $40 \%$ was mandatory.

The work plan was discussed with multidisciplinary team including (hepatic surgeon, hepatologist, medical oncologist \& interventional radiologist). Management of multiple colorectal liver metastases entails the use of other therapeutic modalities before surgery. The general principle of these modalities is to increase the residual liver volume and convert the unresectable metastases to resectable ones. The final aim was to achieve complete (R0) resection of all the liver metastases. In all cases of metachronus liver metastases and some cases of synchronous liver metastases which were irresectable with the primary tuomrs, neoadjuvant chemotherapy was started before planned hepatectomies. The benefits of neoadjuvant therapy were to convert unresectable lesion to resectable one and to test biological behavior of the tumors. Any progression on chemotherapy was considered a contraindication for surgery. Portal vein embolization was done when FLR was inadequate and reassessment with volumetric CT was done after 4 weeks. Portal vein ligation was done in synchronous lesion with inadequate FLR during resection of the primary and reassessment with volumetric CT was done after 4 weeks.

In synchronous metastases, combined resection should be planned when possible but it is not recommended in urgent colonic 
resection, locally advanced colorectal carcinoma (CRC) and cases demanding a major hepatic resection.

\section{Operation:}

Patients were positioned supine. All cases were done via laparotomy. Either a hockey stick incision or midline incision was used (xiphisternal extension). Subcostal retractors were used to provide strong retraction of the costal margin. The laparotomy began with a thorough examination of the peritoneal cavity to exclude any peritoneal disease or pelvic recurrence (when possible). All regional lymph nodes (porta-hepatis and coeliac) were assessed and any suspicious nodes were sent for a frozen section examination. Bimanual palpation of the liver combined with intra-operative US examination of the liver allowed confirmation of the number, size and position of each metastasis and helped to identify lesions missed on pre-operative imaging.

Liver resection was either anatomical resection, in which one or more complete segments were removed or atypical (wedge) resection. The type of resection performed depended on size, site, number of tumors and relation to vascular and biliary structures. Small superficially located tumors were resected by wedge resection or 'metastasectomy'. Safety margins of $10 \mathrm{~mm}$ were recommended but safety margins of $5 \mathrm{~mm}$ were accepted. Larger and multiple lesions usually needed anatomical resection. We used the Harmonic Scalpel (Ethicon Endo-Surgery) for liver parenchyma resection.

Patients who presented with a technically 'easily' resectable primary tumour and peripherally placed low-volume liver disease were amenable to synchronous resection of both primary and metastatic liver disease at the same procedure, without increased morbidity or mortality. Those patients who presented with large bowel obstruction and synchronous CRLM had immediate definitive treatment for their life-threatening colonic emergency (endoscopic stenting, resection with either a stoma or immediate reconstruction). When resection of the primary tumour was more demanding, when treatment of the primary required neoadjuvant treatment or when the liver disease was such an extent that at least a hemi-hepatectomy or more was required, a planned sequential staged procedure was preferred.

\section{Pathological assessment:}

All specimens were examined histopathologically to confirm diagnosis of metastatic colorectal carcinoma, accurate assessment of number, site, size (largest), grade, macroscopic or microscopic vascular invasion and safety margin.

Post-operative care and follow up:

Early post-operative, patients were admitted to intensive care unit (ICU) especially if major hepatectomy was done to ensure close monitoring and adequate analgesia. Blood tests and serologic liver function tests were performed during the $1^{\text {st }}, 3^{\text {rd }}, 5^{\text {th }}$ and $7^{\text {th }}$ days after resection. All patients underwent an Xray chest examination on the $3 \mathrm{rd}$ postoperative day, and ultrasonography was performed during the first week after resection. CT abdomen was done when complications were expected and the US failed to diagnose.

After discharge, patients were assessed 4 weeks later. In this visit, in addition to physical examination, liver functions and tumor markers were tested. A return of tumor markers to normal (if previously elevated) served to confirm that the goal of resection has been achieved. If they did not return to normal, a meticulous search for treatable tumor was performed.

Following this postoperative check, patients were seen at 3, 6 and 12 months after surgery. During each of these visits liver functions and tumor markers were assessed and a CT scan of the chest, abdomen and pelvis were performed. Suspicions raised by patient symptoms, CT findings or elevated tumor marker required further investigation by MRI or FDG-PET scans.

\section{Results:}

This study was conducted on 30 patients with multiple colorectal liver metastases. They included 16 males $(53.3 \%)$ and 14 females $(46.7 \%)$. The mean age for this group study was 49.9 years (ranging from 27 to $75 y$ ) with $\mathrm{SD} \pm 10.31$. The mean follow up period was 12 months. 
Criteria of primary colorectal tumors:

Pathological analysis of the colorectal specimens revealed the following: 28 cases were adenocarcinoma (93.3\%), 1 mucinous adenocarcinoma (3.3\%) and 1 liomyosarcoma of the colon $(3.3 \%)$. All specimens were pathologically confirmed with free safety margins.

LNs involvement of the resected colonic samples was positive in $76.7 \%$ of cases $(n=23)$ and negative in $23.3 \%$ of cases $(n=7)$.

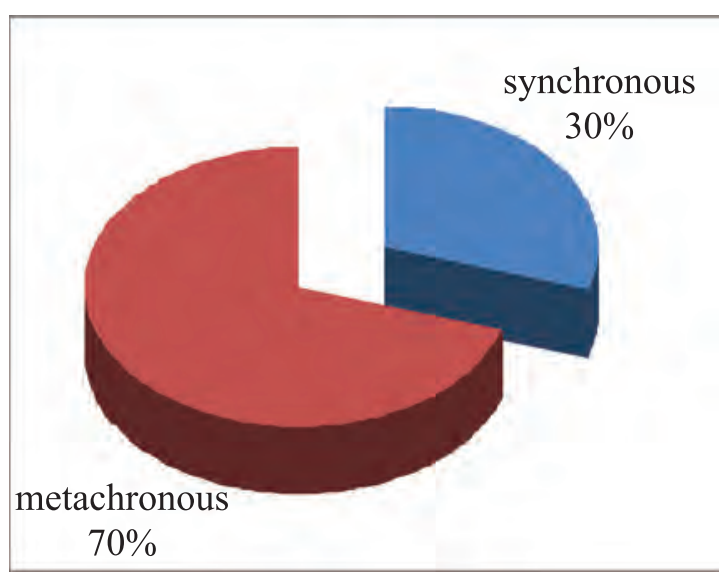

Figure (1): Presentation of colorectal liver metastases.

The primary operative management was in the form of major hepatic resection in 50\% $(n=15)$ of cases (right hepatectomy in 5 cases, left hepatectomy in 3 cases and central hepatectomy in one case. Combination procedures of right posterior hepatectomy with

\section{Criteria of metastatic liver lesions:}

Nine patients (30\%) had synchronous, and 21 patients $(70 \%)$ had metachronus colorectal liver metastases Figure(1). Ninety one colorectal metastatic tumors were identified with a mean size of $4.3 \pm 2.5 \mathrm{~cm}$ (ranging from 2 to $12 \mathrm{~cm}$ ); lesions were situated in the right, left lobes and bilobar in 53\%, 12\%, and 35\% of the cases respectively Figure(2).

Pre-operative assessment of CEA and CA19.9 showed the following results, 14 patients out of the $30(47 \%)$ patients had elevated serum levels of CEA and 7 cases $(23 \%)$ had elevated serum levels of CA19.9.

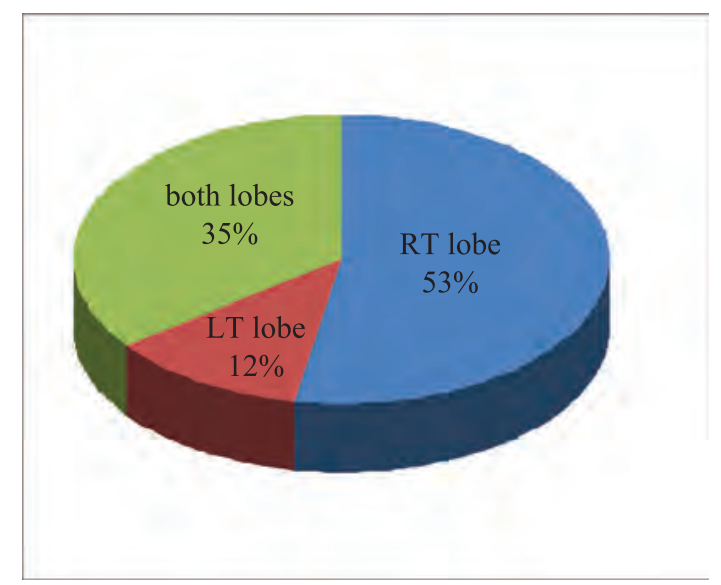

Figure (2): Distribution of liver metastases.

left lateral hepatectomy was done in 3 cases; combination of left lateral hepatectomy with segmentectomy in 3 cases). Bisegmentectomy was done in $27 \%(\mathrm{n}=8)$, and segmentectomy in $23 \%$ of cases $(n=7)$ Figure(3).

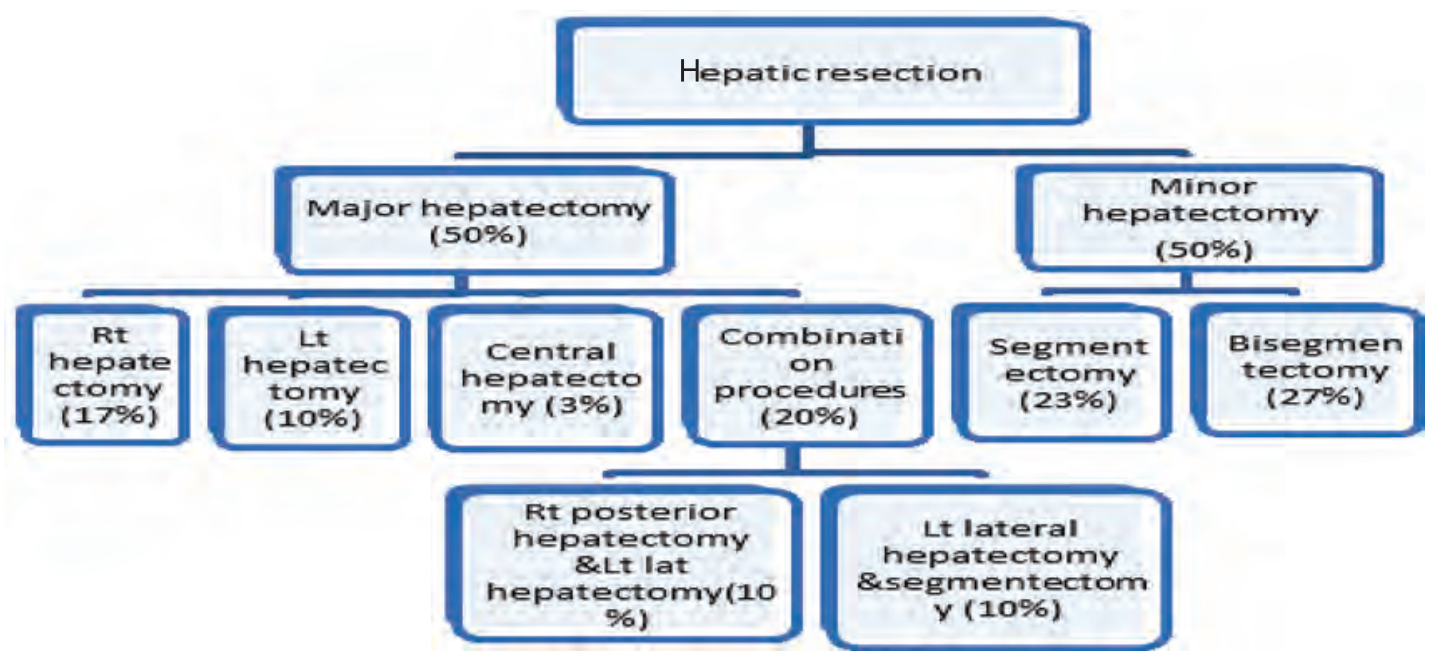

Figure (3): Algorithm for hepatectomies done in this study. 


\section{Synchronous tumors:}

Nine cases $(30 \%)$ presented with synchronous hepatic metastases. Four cases (13.3\%) underwent resection only for the colorectal malignancy in the first laparotomy due to colonic cause like obstruction or hepatic cause like inadequate FLR. Those patients got adjuvant chemotherapy to downstage the liver metastatic disease then underwent hepatic resection.

5 cases $(16.7 \%)$ of them underwent surgical intervention for the hepatic metastases synchronously. Three cases (10\%) underwent definitive hepatic resection and 2 patients
$(6.7 \%)$ with bilobar liver metastases with inadequate future liver remnant were planned for 2 staged hepatectomies. Both had radical local resection of the primary tumor. One case had intraoperative right portal vein ligation and the other, underwent right portal vein ligation in association with non-anatomical metastatectomy for left lobar metastatic lesions. In the second stage operation, one of the 2 cases had right hepatectomy with $\mathrm{R} 0$ resectability and adequate liver remnant volume, while the other was deemed irresectable Figure(4).

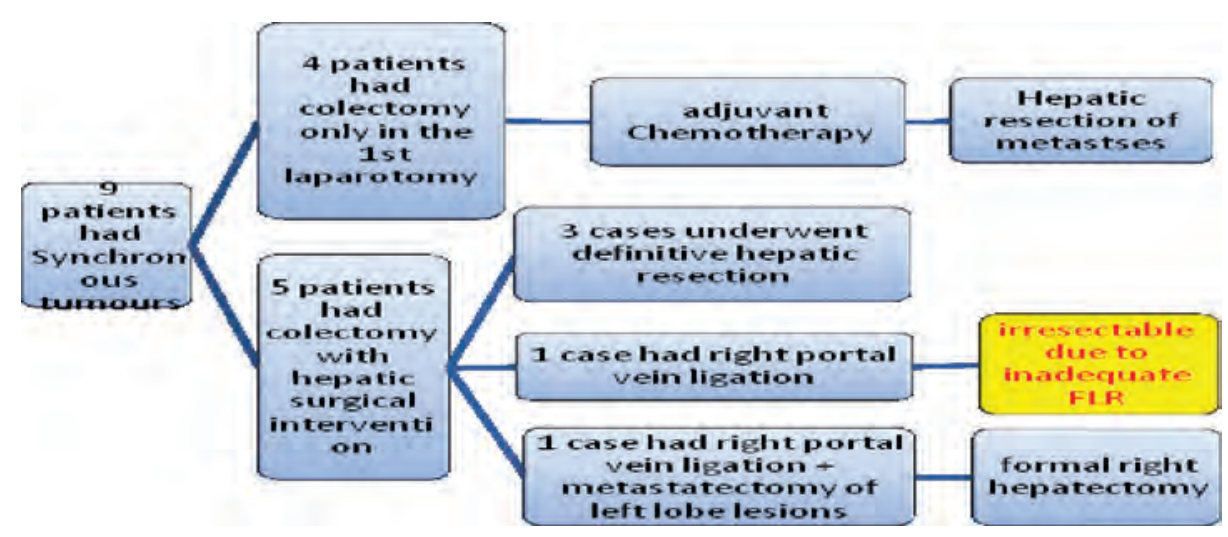

Figure (4): Algorithm of management of synchronous metastases.

\section{Metachronous tumors:}

Twenty one cases $(70 \%)$ presented with metachronous hepatic metastatases. The tumor free survival was $20.7 \pm 15.7$ months ( 5 to 60 months). All of them had received preoperative adjuvant chemotherapy with good response in $60 \%(n=18) .3$ patients $(10 \%)$ shifted to locally ablative therapy due to unavoidable side effects of systemic chemotherapy. Transcatheter Arterial Chemoembolization (TACE) was used successfully in 2 cases (7\%) and ultrasound guided Radiofrequency Ablation (RFA) in one case $(3 \%)$ with considerable decrease in size of hepatic metastases then followed by hepatectomy Figure(5).

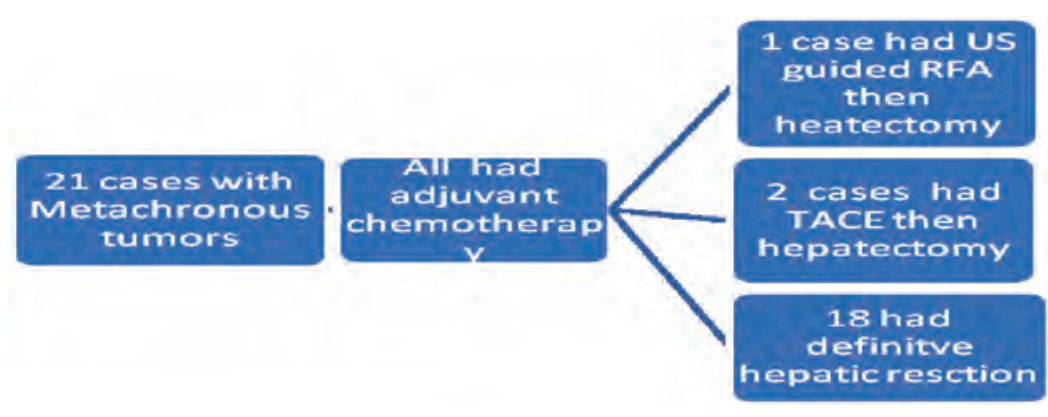

Figure (5): Algorithm of management of metachronous metastases. 


\section{Post-operative hospital stay:}

The mean postoperative hospital stay was $9.5 \pm 3.13$ days (ranging from, 7 to 23 days). Twenty nine cases required postoperative ICU admission (97\%) to achieve strict monitoring

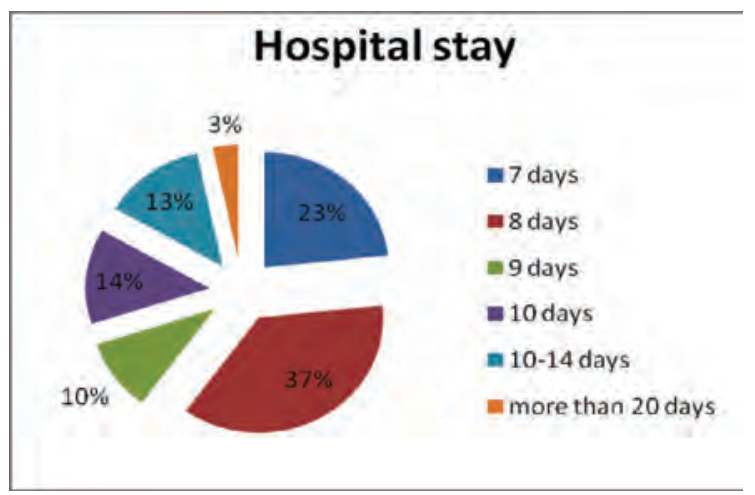

Figure (6): Postoperative hospital stay.

Early postoperative complications:

Early postoperative complication was considered with any complication occurring within 30 days from the operation. Six patients $(20 \%)$ had postoperative complications, the most common one was biliary leak which occurred in three cases (10\%). Conservative management in the form of maintained external drainage with antibiotics according to culture and sensitivity (C\&S) was effective in the management of all 3 cases. Intra-abdominal collection occurred in one case $(3 \%)$ on the $7^{\text {th }}$ postoperative day and was managed by ultrasound guided pig-tail external drainage and early detection of any complication. The mean postoperative ICU stay was $1.8 \pm$ 1.04days (ranging from, 0 to 6 days) Figures(6,7).

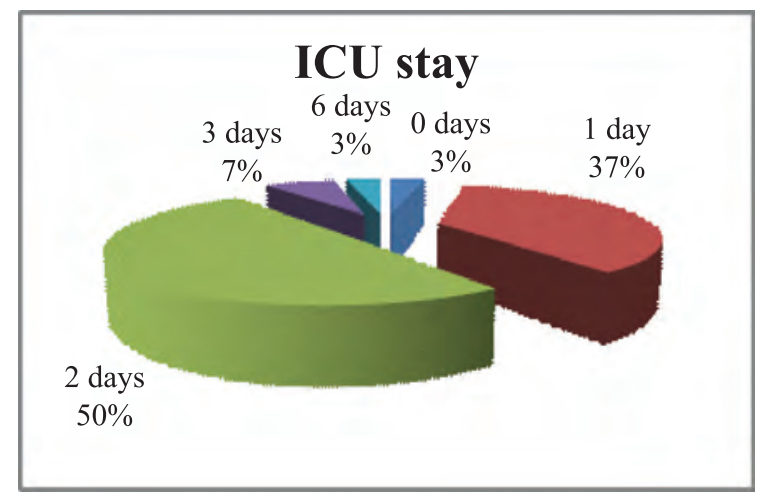

Figure (7): Postoperative ICU stay.

together with antibiotics according to culture and sensitivity. Chest infection was reported in one case (3\%) that was successfully treated with antibiotics according to sputum $C \& S$. Pulmonary embolism occurred in one case $(3 \%)$, it was controlled effectively with conservative measures and anticoagulant therapy with low molecular weight heparin during the hospital stay and then shift to oral warfarin to maintain INR between 2 and 3 for 12 months postoperative. No cases were complicated by postoperative liver cell failure. Mortality during early postoperative period was 0 Figure(8).

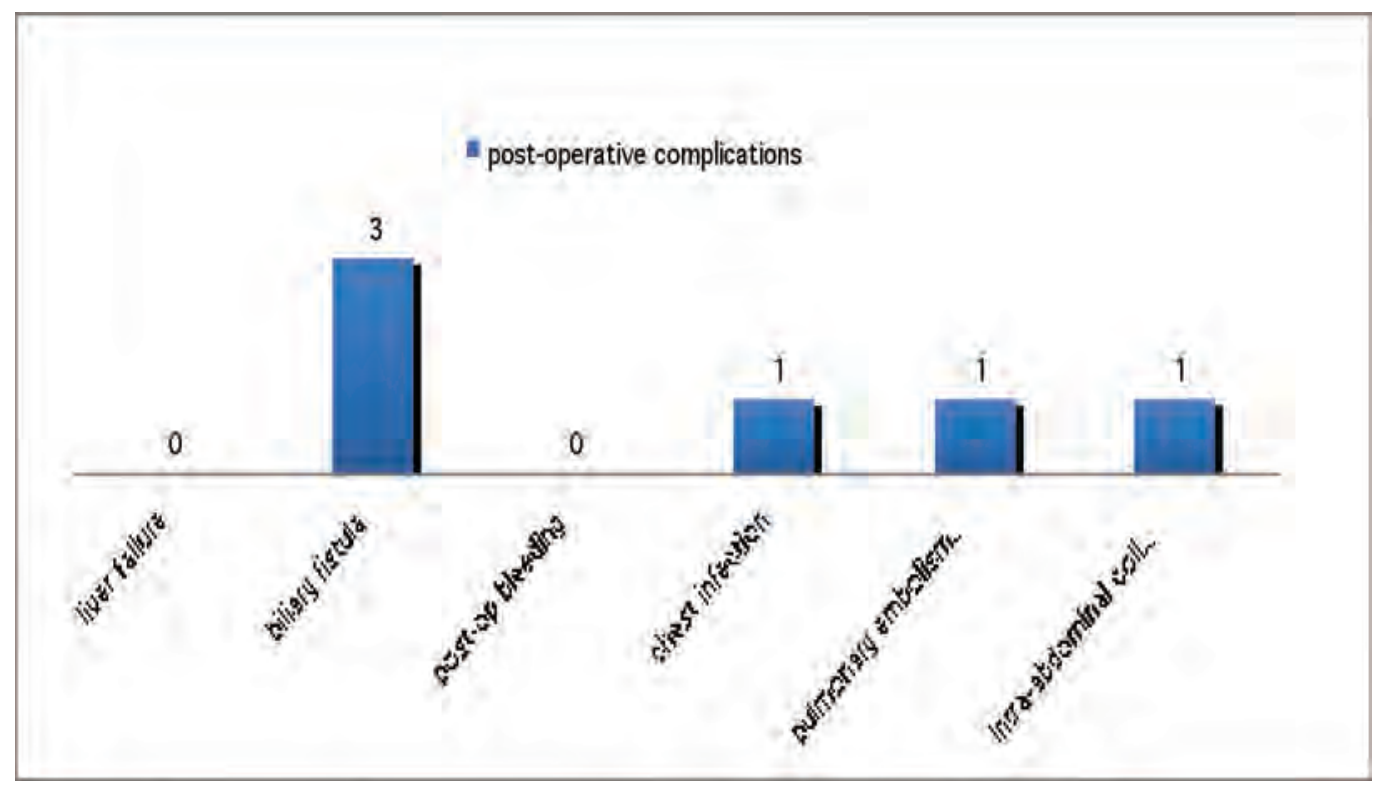

Figure (8): Post-operative complications. 


\section{Recurrence:}

Mean follow up period was $12 \pm 8$ (ranging from 3 to 37 months). Nine patients $(30 \%)$ had recurrent malignant disease, the recurrence was local, hepatic or distant extra-hepatic. Hepatic recurrence occurred solely in 2 cases $(6 \%)$. One case was managed by hepatic resection and the other by percutaneous RFA. Recurrence of colorectal carcinoma (local) occurred in 5 cases $(17 \%)$. Combined local and distant extra-hepatic lesions in 2 cases $(6 \%)$; both were managed by chemotherapy with or without radiotherapy Figure(9).
Eight cases out of the nine recurrent cases showed re-elevation of serum level of CEA. The two cases of hepatic recurrence showed fast rise of serum CEA.

\section{Mortality:}

A single mortality case was recorded during the follow-up period of 12 months, with a 1year survival of $97 \%$. This case passed the early post operative period and started to receive chemotherapy, she died from extensive myocardial infarction 3 months after the surgery.

Another case of mortality had been recorded 15 months postoperatively due to respiratory failure from pulmonary metastases.

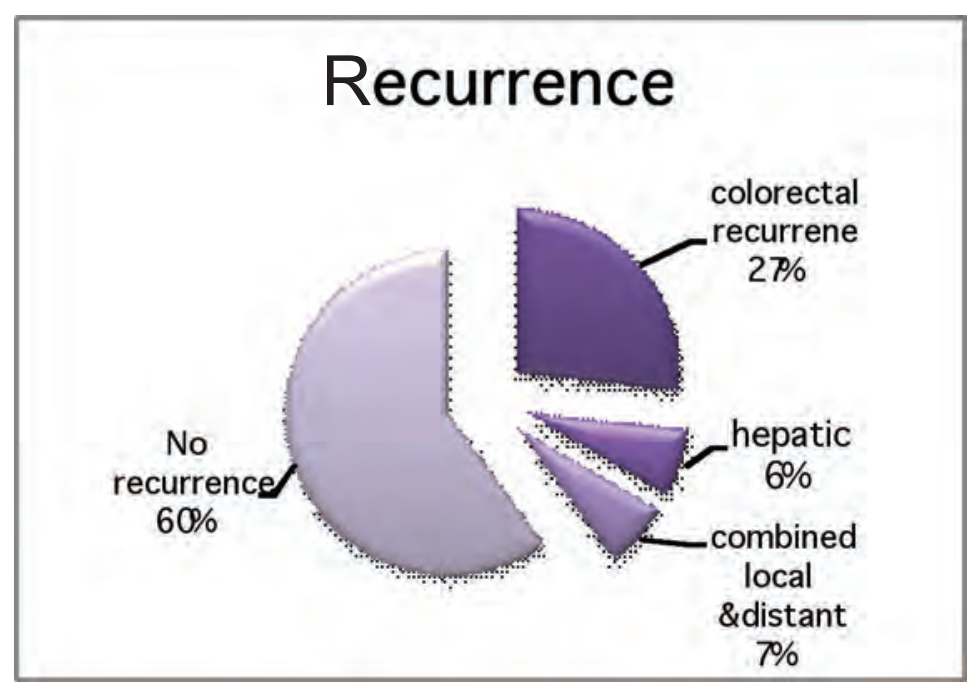

Figure (9): Distribution of recurrence.

\section{Discussion:}

Colorectal cancer accounts for the third most common malignancy in western countries. More than $75 \%$ of all patients will develop hepatic metastases during the course of disease; up to $25 \%$ of them will have liver metastases at diagnosis. ${ }^{5}$ Malignant tumors of the liver were once considered a lethal disease. Untreated metastatic colorectal carcinoma has a median survival of 6 to 9 months. ${ }^{6}$

Improved perioperative outcomes for hepatic resection have resulted in part from a shift from a focus on "what comes out" (ie, tumors) to "what stays in" (ie, liver). Similarly, resectability is no longer restricted based on the number, size, or unilaterality of tumors when an adequate liver remnant will remain after complete resection of tumor-bearing liver. As a result, patients with more and larger lesions undergo resection, including patients who require extensive resection of liver parenchyma, yet durable survival is achieved. ${ }^{7}$

Based upon pre- and peri-operative assessment, patients are candidates for surgery if they have no non-resectable extrahepatic disease, all liver deposits can be resected with tumour-free margins, and sufficient liver parenchyma can be preserved to avoid postoperative liver insufficiency. Surgical resection is, to date, the only potentially curative treatment of colorectal metastases. ${ }^{8}$

No existing treatment other than surgery can result in long-term survival, but only $10-20 \%$ of patients with liver metastases fulfill standard selection criteria and are amenable to surgery. As a consequence, the trend is to be more aggressive and to increase the indications for surgical resection. Portal vein embolization, ablative techniques and chemotherapy may render patients amenable 
to surgery patients who would have been refused some years ago. ${ }^{8}$

In this study, 30 patients presented with multiple colorectal liver metastases underwent surgical intervention (33 successful hepatectomies) with curative intent. The surgery was used as the main line of treatment in all cases; accompanied with other therapeutic modalities when needed. The patients were followed up postoperatively for a median of 12 months to assess the outcome of this approach in terms of incidence of morbidities and mortalities in the study period.

The goal of surgery for liver metastases is to remove all the metastatic sites, if possible with a free clearance margin of $1 \mathrm{~cm}$. The type of liver resection depends on the size, the number, and the location of the metastases, as well as their relation to the main vascular and biliary pedicles and the volume of the liver parenchyma that can be left in place after surgery.

In some cases, the choice between performing several wedge resections or a major liver resection removing all the deposits at once can be difficult. The first solution preserves more healthy liver parenchyma, but the cut section of the liver may be larger increasing the risk of postoperative hemorrhage or fluid collection. On the other hand, a major liver resection allows a better clearance between tumour deposits and the cut section of the liver, a better control of peri-operative haemorrhage and the recognition of main intrahepatic vessels, but removes more parenchyma with a risk of post-resection hepatic failure and the theoretical risk of promoting the development of dormant liver metastases by the mechanisms involved in liver regeneration. In addition, a large resection may preclude further treatment in case of intrahepatic recurrence.

Surgeons generally prefer good margins around vital structures, preferably $10 \mathrm{~mm}$. However, it has been known for a while that any margin at all will actually suffice as most liver metastases are 'pushing' rather than infiltrative. ${ }^{9}$

Synchronous colorectal liver metastases:

Synchronous colorectal liver metastasis is found in $20 \%$ to $30 \%$ in patients at the time of initial diagnosis. Surgical management of this group of patients is controversial and widely debated. Some authors have reported higher complication and mortality rates for patients with simultaneous resections. Nordlinger and colleagues reported an operative mortality of $7 \%$ of simultaneous resection, compared with $2 \%$ with staged resection. A staged operative approach was therefore recommended, with the liver resection performed 2 to 3 months after the resection of the primary. The increase in complications in case of staged resection group may be explained by the need for two laparotomies. ${ }^{10}$

Simultaneous resection of the primary colon tumor and hepatic metastasis is safe and efficient in treating patients with synchronous colorectal metastasis. The overall complication rate is lower for simultaneous resection than that for staged resection, mortality rate is the same, and the length of hospital stay is significantly less. Because it avoids a second laparotomy and reduces complications; simultaneous resection of the colon primary tumor and liver metastases should be considered the preferred treatment for suitable patients with resectable synchronous colorectal metastasis. Because effective adjuvant therapies exists for colorectal cancer, a simultaneous resection also provides for prompt completion of surgical therapy and earlier initiation of adjuvant therapy in this group of patients with high risk of additional microscopic diseases who are most likely to benefit from such adjuvant therapy. ${ }^{11}$

Many authors suggest that if a synchronous hepatic lesion is small, peripherally located, and easily removed at the time of primary resection with negligible increase in the morbidity or mortality of the surgery, it is best to do so. In the fortunate circumstance that this proves to be the patient's only hepatic lesion, this would spare the morbidity of a second laparotomy after an interval reevaluation. ${ }^{12}$

In this study, 9 cases of synchronous liver metastases were operated on. They were investigated and assessed well. Five of them underwent surgical intervention for the hepatic metastases with the colectomy in the same laparotomy, 3 cases underwent definitive hepatic resection and two cases of synchronous colorectal liver metastases had their first step 
of surgical hepatic management during the first laparotomy. First, had left lateral hepatectomy and right portal vein ligation, the other case had portal vein ligation alone.

Methods of increasing resectability:

One of the main causes of CRLM irresectability was technically irresectable tumor (impossible to achieve a disease-free margin or insufficient hepatic reserve to support postoperative hepatic function). The following strategies may be undertaken to allow a potentially curative resection for these patient groups.

\section{Adjuvant chemotherapy:}

The aim of downsizing chemotherapy is to reduce the size of the tumors. In general, the rationale for using this therapy in the context of irresectable disease is clear, with overall survival in patients receiving systemic chemotherapy but not surgery reaching approximately 2 years. ${ }^{13}$ The potential to downsize disease, and to convert irresectable disease to resectable, is even more intriguing. Adam et al ${ }^{14}$ reported being able to achieve downsizing to enable resection in $13 \%$ of 1,104 patients initially having irresectable metastases, with good outcomes (5-year overall survival of $33 \%$ and 5 -year disease free survival of $22 \%$ ); this population included patients having extrahepatic disease. Results achieved with downsizing through chemotherapy for initially irresectable liver-only disease are even more encouraging, with up to $44 \%$ of patients being able to proceed to complete resection after chemotherapy. ${ }^{15}$ Although chemotherapy can cause liver toxicity, numerous studies have shown that hepatic resection after chemotherapy is safe in properly selected patients. Although few reports suggest that intra-arterial therapy may lead to downsizing to enable resection, hepatic arterial infusion (HAI) has not been used widely, possibly due to technical issues with regard to delivery of the therapy. As salvage for systemic chemotherapy failure, HAI holds some promise. ${ }^{16}$

In this study, $83 \%$ of the cases received downsizing chemotherapy. Ninety two percent of those patients who received downsizing chemotherapy responded well with considerable decrease in the size of the metastases and increase in the anticipated future liver volume These results were biased by patients' selection due to exclusion of nonresponding patients,

\section{Two-stage resection:}

Adam et al. have suggested using two-stage hepatectomy to convert non-resectable liver metastases into potentially curable cases. This strategy is usually applied to multinodular bilobar metastases. The first-stage resection is intended to remove the highest possible number of tumour lesions. This is followed by a liver regeneration period. During this period the patient is usually treated with chemotherapy to limit disease growth. It is recommended that this chemotherapy should start 3 weeks after surgery (so that it does not interfere with initial regeneration) and continue for 2-3 months, when a further set of assessment tests are performed in order to evaluate the patient for the second curative stage. The second stage is only performed if it is potentially curative and only if enough parenchymal hypertrophy has occurred to reduce the risk of postoperative liver failure. Furthermore, in order for the patient to be eligible for two-stage hepatectomy, the tumour should be downstaged or stabilized by initial neoadjuvant chemotherapy. The objective of this technique is to avoid posthepatectomy liver failure. Long-term results from this strategy are still awaited, but initial evaluation of a small series suggested a 3 year survival of $35 \%$ and a perioperative mortality risk of $15 \% .{ }^{17}$

In this study, 2 patients with synchronous multiple, bilateral CRLM with inadequate future liver remnant were planned for 2 staged hepatectomies; in the first stage, both had radical local resection of the primary tumor; one case received intraoperative right portal vein ligation, the other, underwent right portal vein ligation in association with non anatomical resection for a Lt lobe metastatic lesions. In the second stage operation, one of the 2 cases had Rt hepatectomy with $\mathrm{R} 0$ resectability and adequate liver remnant volume, while the other was deemed irresectable.

The operative mortality for major hepatic resections has declined with improved operative techniques and postoperative care, but morbidity remains significant. Operative 
mortality ranged from $0 \%$ to $7 \%$, and causes of death included hemorrhage, sepsis, and hepatic failure. Morbidity was between 22\% and $39 \%$, and common causes of morbidity included hemorrhage, biliary leak or fistula, hepatic failure, perihepatic abscess, wound infection, pneumonia, and myocardial infarction. 18

In our study, this was no cases of perioperative mortalities, that may be due to proper patients selection depending on MDT decision.

As regard morbidities, (20\%) of this study group patients had major postoperative complications, the most common one was the biliary leak which occurred in three cases $(10 \%)$, they were diagnosed clinically and conservative treatment was successful in the 3 cases. Intra-abdominal collection occurred in one case (3\%) that was treated by ultrasound guided aspiration under umbrella of antibiotics, chest infection in one case $(3 \%)$ that was successfully treated with antibiotics and pulmonary embolism in one case $(3 \%)$ that was treated conservative with anticoagulant therapy (starting with low molecular weight heparin then oral warfarin for 1 year). No cases of posthepatectomy liver failure occurred, this might be due to proper assessment of risky patients by volumetric liver studies, using neoadjuvant chemotherapy to decrease the size of metastases and applying surgical techniques like portal vein ligation and two staged hepatectomies in selected cases.
Recurrence can occur in as many as $60 \%$ of patients following liver resection of colorectal metastatic disease, with the most frequent site of recurrence being the liver. In approximately $20 \%$ of these patients the liver may be the only site of recurrence and as a result these patients may be suitable for reresection. The vast majorities of these recurrences occur in the first two years and for that reason frequent surveillance with $\mathrm{CT}$ is critical for early detection. This becomes even more important if we consider that the reported morbidity and mortality rates, as well as overall survival rates after re-resection, are similar to those reported for the initial hepatectomy, despite the potentially greater technical difficulty. In the current cost conscious environment, the fact that intensive 3-monthly CT surveillance detects recurrence that is amenable to further resection in a considerable number of patients, leads to significantly better survival for these patients with a reasonable cost per life-year gained. ${ }^{19}$

In our study, the incidence of recurrence was $30 \%$. Nine patients had recurrent malignant disease, the recurrence was local, hepatic or distant extra-hepatic. Recurrence was distributed as the following; local recurrence of colorectal carcinoma occurred in $18 \%$ of cases; hepatic recurrence occurred in $6 \%$ of cases (one managed by re-hepatic resection and the other by RFA) and combined both local and distant extra-hepatic lesions in 6\% of cases. These cases were managed by chemotherapy.

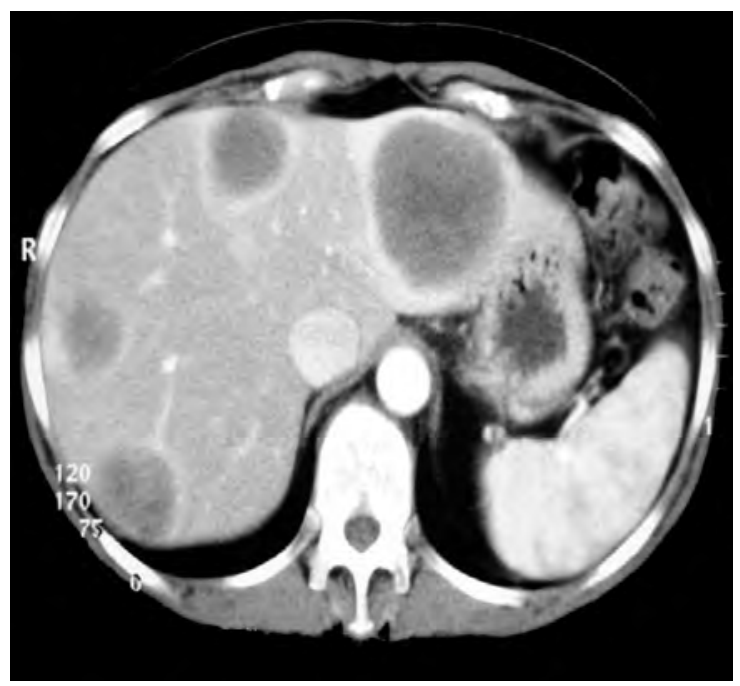

Figure (10): Multiple colorectal liver metastases. 


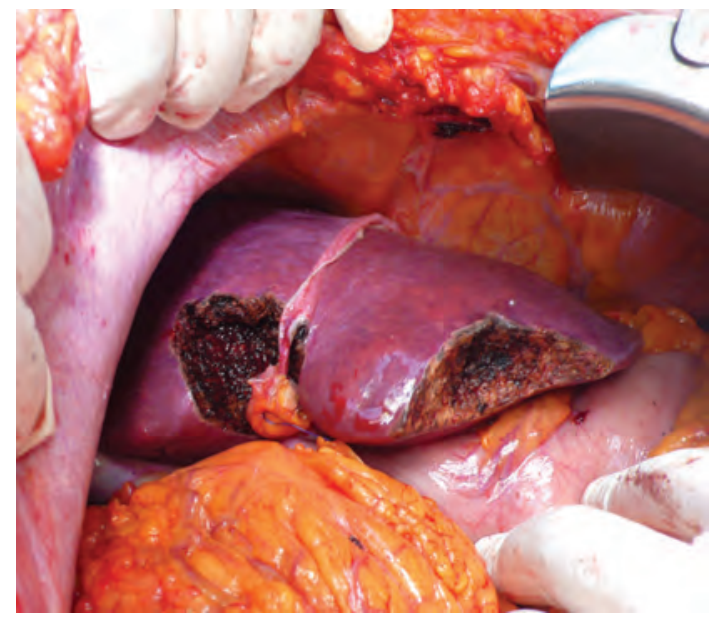

Figure (11): Synchronous colorectal liver metastases. This patient had left hemicolectomy + resection of 2 metastases in segments III \& IVa+ ligation of Right PV.

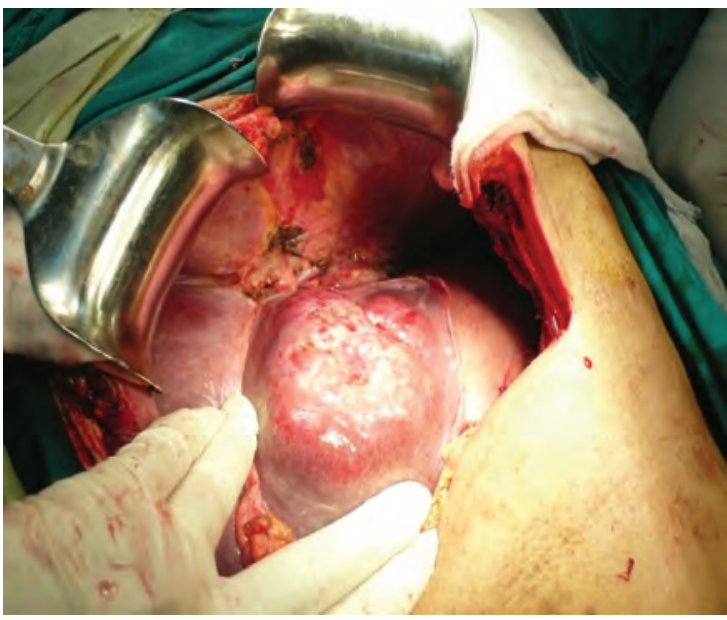

Figure (12): Left lobe liver metastases. This patient had other metastases in segment 7 in the right lobe.

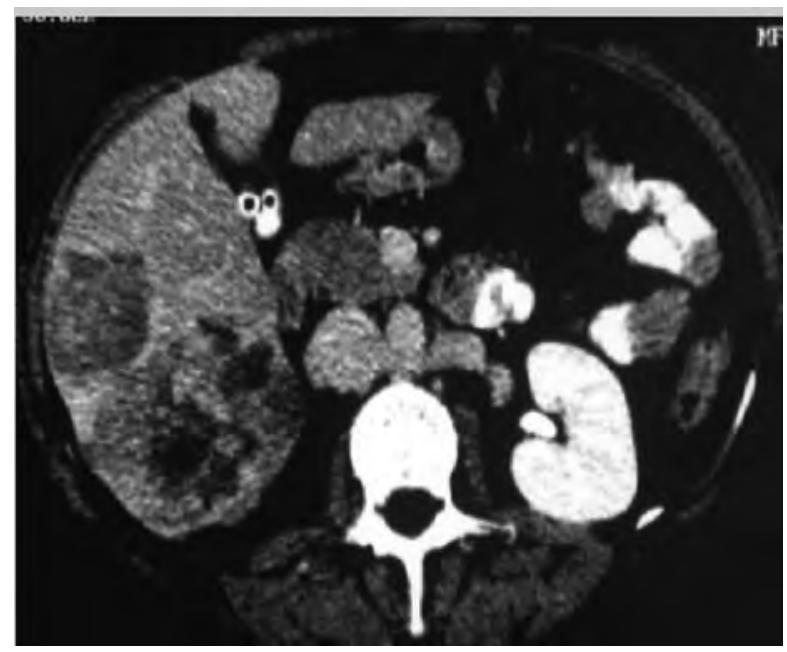

Figure (13): CT scan showing multiple colorectal liver metastases.

\section{References:}

1- Reinhart T Grundmann: Current state of surgical treatment of liver metastases from colorectal cancer. World J Gastrointest Surg 2011; 3(12): 183-196.

2- Evangelos P Misiakos, Nikolaos P Karidis, Gregory Kouraklis: Current treatment for colorectal liver metastases. World $J$ Gastroenterol 2011; 17(36): 4067-4075.

3- Viganò L, Ferrero A, Lo Tesoriere R, Capussotti L: Liver surgery for colorectal metastases: Results after 10 years of followup. Long-term survivors, late recurrences, and prognostic role of morbidity. Ann Surg Oncol 2008; 15: 24582464.

4- Pulitanò C, Castillo F, Aldrighetti L, Bodingbauer M, Parks RW, Ferla G,
Wigmore SJ, Garden OJ: What defines 'cure' after liver resection for colorectal metastases? Results after 10 years of followup. $H P B$ (Oxford) 2010; 12: 244-249.

5- Taylor I: Liver metastases from colorectal cancer: Lessons from past and present clinical studies. Br J Surg 1996; 83: 456460.

6- Khatri VP: Metastatic colorectal carcinoma: pushing the surgical envelope of cure. Ann Surg Oncol 2005; 12: 866-867.

7- Abdalla EK: Surgical management of colorectal liver metastases. Community Oncology. Volume 6/Number 8.2009.

8- Penna C, Nordlinger B: Surgery of liver metastases from colorectal cancer: New promises; British Medical Bulletin 2002; 64: 127-140. 
9- de Haas RJ, Wicherts DA, Flores E, Azoulay $\mathrm{D}$, Castaing D, Adam R: R1 resection by necessity for colorectal liver metastases: Is it still a contraindication to surgery? Ann Surg 2008; 248: 626-637.

10-Nordlinger B, Jaeck D, Guiget M: Surgical resection of hepatic metastasis, multicentric retrospective study by the french association of surgery. In: Treatment of hepatic metastases of colorectal cancer. Nordlinger B, Jaeck D (Editors); New York Paris, Springer Verlag (Publisher); 1992; p. 129146.

11-Martin R, Paty P, Fong Y: Simultaneoues liver and colorectal resections are safe for synchronous colorectal liver metastasis. American College of Surgeons 2003.

12-Lambert A, Thomas A, Colacchio: Interval hepatic resection of colorectal meastases imoroves patient selection. Arch Surg 135 2000; 473-480.

13-Zorzi D, Laurent A, Pawlik TM, Lauwers GY, Vauthey JN, Abdalla EK: Chemotherapy- associated hepatotoxicity and surgery for colorectal liver metastases. Br J Surg 2007; 94: 274-286.

14-Adam R, Pascal G, Castaing D, Azoulay D, Delvart V, Paule B, et al: Tumor progression while on chemotherapy: A contraindication to liver resection for multiple colorectal metastases? Annals of
Surgery 2004; 240(6): 1052-1061 [discussion 1054-61].

15-Alberts SR, Horvath WL, Sternfeld WC, et al: Oxaliplatin, fluorouracil, and leucovorin for patients with unresectable liver-only metastases from colorectal cancer: A North Central Cancer Treatment Group phase II study. J Clin Oncol 2005; 23: 9243-9249.19.

16-Boige V, Malka D, Elias D, et al: Hepatic arterial infusion of oxaliplatin and intravenous LV5FU2 in unresectable liver metastases from colorectal cancer after systemic chemotherapy failure. Ann Surg Oncol 2008; 15: 219-226.

17-Adam R, Delvart V, Pascal G, et al: Rescue surgery for unresectable colorectal liver metastases downstaged by chemotherapy: A model to predict long-term survival. Ann Surg 2004; 240: 644-657.

18-Fong Y, Cohen AM, Fortner JG, et al: Liver resection for colorectal metastases. $J$ Clin Oncol 1997; 15: 938-946.

19-Gomez D, Sangha VK, Morris-Stiff G, Malik HZ, Guthrie AJ, Toogood GJ, Lodge JP, Prasad KR: Outcomes of intensive surveillance after resection of hepatic colorectal metastases. Br J Surg 2010; 97: 1552-1560. 